\title{
Seed storage, temperature and relative humidity: response
}

Recently, we introduced the idea of using thermodynamic principles to model the nature and kinetics of seed aging (Vertucci and Roos, 1990), which has stimulated considerable interest (Ellis et al., 1991; Vertucci and Roos, 1991; Smith, 1992). The recent correspondence by Smith (1992) deserves some further comments regarding the thermodynamic principles and their implications for seed storage protocols.

Smith (1992) makes a distinction between the 'threecomponent' system (seed, air and a saturated salt solution) that we use in our experiments (Vertucci and Roos, 1990) and the 'two-component' system (seed and air) used by others (for example, Tompsett, 1986; Ellis et al., 1989, 1990), and questions the validity of comparing the two systems. He suggests that there is no information about the partition of water in seeds versus the surrounding air. This is, in fact, not the case. That partition is described by moisture isotherms: curves that describe the unique relationship between water content, relative humidity and temperature when the system is at equilibrium. Moisture sorption isotherms for a variety of seeds at various temperatures have been described previously (for example, Vertucci and Leopold, 1987; Vertucci and Roos, 1990, 1991). Moisture isotherms of air at various temperatures are described by psychrometric charts available in humidity control manuals (e.g. Harriman, 1990). Smith (1992) suggests that the initial moisture content and temperature of seeds affect the subsequent shape of moisture sorption isotherms. However, because isotherms are representative of the equilibrium condition, we know that the temperature/relative humidity/moisture content relationships are independent of seed or air volume or the initial conditions (Atkins, 1982).

Smith's comments were made in the context of what happens when temperature is changed. Le Chatelier's Principle tells us that if a system at equilibrium is perturbed (i.e. temperature is changed), the system will change to restore equilibrium: in other words, the equilibrium conditions will change (Atkins, 1982). In a three-component system, the relative humidity of the air is buffered by the salt solution, and so it changes only slightly with temperature, and these changes are available in published tables. Moisture sorption isotherms document that when temperature is lowered and relative humidity is constant, the moisture content of seeds increases (Vertucci and Leopold, 1987; Vertucci and Roos,
1991). This is the opposite of what is expected for air according to psychometric charts. The difference between the response of seed moisture content and air moisture content to temperature change is because vapour condensation (air to seed) is an exothermic process and vaporization (seed to air) is an endothermic process. According to Le Chatelier's Principle, a reduction in temperature favours the products for exothermic reactions and the reactants for endothermic reactions (Atkins, 1982).

We disagree with the statement by Smith (1992) that "the 'two-component' system is more appropriate [than a buffered system] for modelling of current seed-bank procedures." Since seeds will be in equilibrium with their environment during long-term storage, it is irrelevant whether or not equilibrium was achieved using a buffered system. The equilibrium conditions can be deduced in a three-component system from moisture sorption isotherms and calculated in a two-component system from moisture sorption isotherms, a psychometric chart and the volume of the seed and air space. If a two-component system is used and the package has limited headspace, the moisture content of the seeds will not change perceptibly. According to moisture sorption isotherms, if seeds at a given moisture content are cooled, the relative humidity decreases. This is what happens when typical seed storage practices are used (Cromarty $e t$ al., 1985). Notice that exactly the opposite effect occurs in many studies designed to determine the optimum moisture content for storage: the temperature of seeds at a given moisture content is raised resulting in a higher relatively humidity. We believe that caution must be exercised when extrapolating data obtained from accelerated aging studies to actual seed storage conditions because the thermodynamic conditions of the two environments may be quite different.

There is no support for the contention made by Smith (1992) that sorption isotherms at different temperatures will approach a single isotherm "influenced by the temperature at which the seeds were [originally] equilibrated' if a package with limited air space is used and the air is unbuffered. If this were true, then the slope of the van't Hoff analysis would equal 0 (as Smith (1992) implies), meaning that the enthalpy of water sorption also equals 0 (since the slope of the $\ln (\mathrm{RH} / 100)$ vs $1 / \mathrm{T}$ plot is equal to the enthalpy of sorption $(\Delta \mathrm{H})$ divided by the gas constant $R$ ). 
Smith's contention is inconsistent with both the second and third laws of thermodynamics, since

$$
\begin{gathered}
\Delta \mathrm{G}=\Delta \mathrm{H}-\mathrm{T} \Delta \mathrm{S} \text { and } \\
-(\delta \mathrm{G} / \delta \mathrm{T})_{\mathrm{p}}=\mathrm{S}>0
\end{gathered}
$$

where $\mathrm{G}$ is Gibbs energy function, $\mathrm{H}$ is enthalpy, $\mathrm{T}$ is temperature, $S$ is entropy and $(\delta \mathrm{G} / \delta \mathrm{T})_{\mathrm{p}}$ is the partial derivative of the change in free energy with a slight change in temperature when pressure is held constant. From Smith's arguments, one must deduce that $\Delta \mathrm{G}=0$ and $\Delta \mathbf{H}=0$ if there is no change in moisture content or relative humidity when the temperature is changed (i.e. no reaction occurred and equilibrium was maintained). Entropy thus becomes undefined. There is a unique relationship between moisture content, temperature and relative humidity at equilibrium. This means that moisture sorption isotherms must be determined for each temperature under consideration. Currently, this is not the practice in several laboratories.

In his correspondence, Smith (1992) stated that we give "no indication of the practical consequences of adopting [our] advice." Smith suggests that according to the viability equations (Ellis and Roberts, 1980), seeds at higher moisture levels will age at faster rates, and so the recommendations by Vertucci and Roos (1990) for optimum seed storage will actually promote more rapid deterioration. In answering this criticism, it is important to point out that extrapolations of the viability equations cannot be used to 'prove' that the recommendations by Vertucci and Roos (1990) are incorrect. The authors of the viability equations have documented that the equations lose their predictive value at high and low critical moisture contents (Ellis et al., 1989, 1990, 1991). The low critical moisture content corresponds to a relative humidity of about $40 \%$ at $65^{\circ} \mathrm{C}$ (Vertucci and Roos, 1991). The equations do not predict the accelerated deterioration that we have observed at $35^{\circ} \mathrm{C}$ when relative humidity is less than about $30 \%$ (Vertucci and Roos, 1990). Thus, it is a false premise to assume that the viability equations work at all moisture content/temperature combinations. The thermodynamic principles that Vertucci and Roos $(1900,1991)$ have invoked are consistent with published empirical data. The problem is that there are no complete data sets of aging rates $v s$ moisture contents at $5^{\circ} \mathrm{C}$ or $-20^{\circ} \mathrm{C}$. These data sets are required to validate both the viability equations and the predictions made by Vertucci and Roos (1990) at typical long-term storage temperatures. Before these data become available, seed bank operators can choose to follow protocols that are based on thermodynamic principles where the assumptions are known and testable, or protocols that are based on empirical studies of aging under conditions that are not reflective of nor validated with data from standard storage conditions.

Given the unprecedented loss of global biodiversity, we do not have the time for long-term storage experiments which will resolve the controversy over how best to store the seeds that we collect. However, we can use sound thermodynamic principles to predict the best storage conditions as well as to achieve those conditions. These ideas are discussed further in a communication in this issue.

\section{References}

Atkins, P.W. (1982) Physical Chemistry. W. H. Freeman, San Francisco.

Cromarty, A.S., Ellis, R.H. and Roberts, E.H. (1985) The design of seed storage facilities for genetic conservation. International Board for Plant Genetic Resources, Rome.

Ellis, R.H. and Roberts, E.H. (1990) Improved equations for the prediction of seed longevity. Annals of Botany 45, 13-30.

Ellis, R.H., Hong, T.D. and Roberts, E.H. (1989) A comparison of the low-moisture-content limit to the logarithmic relation between seed moisture and longevity in twelve species. Annals of Botany 63, 601-611.

Ellis, R.H., Hong, T.D. and Roberts, E.H. (1991) Seed moisture content, storage, viability and vigour. Seed Science Research 1, 275-279.

Ellis, R.H., Hong, T.D., Roberts, E.H. and Tao, K.L. (1990) Low-moisture-content limits to relations between seed longevity and moisture. Annals of Botany 65, 493-504.

Harriman, L.G. (1990) The Dehumidification Handbook. Munters Cargocaire, Amesbury, Massachusetts, USA.

Smith, R.D. (1992) Seed storage temperature and relative humidity. Seed Science Research 2, 113-116.

Tompsett, P.B. (1986) The effect of temperature and moisture content on the longevity of seed of Ulmus carpinifolia and Terminalia brassii. Annals of Botany 57, 875-883.

Vertucci, C.W. and Leopold, A.C. (1987) Water binding in legume seeds. Plant Physiology 85, 224-231.

Vertucci, C.W. and Roos, E.E. (1990) Theoretical basis of protocols for seed storage. Plant Physiology 94, 1019 1023.

Vertucci, C.W. and Roos, E.E. (1991) Seed moisture content, storage, viability and vigour. Seed Science Research 1, $277-279$

Christina W. Vertucci and Eric E. Roos United States Department of Agriculture

Agricultural Research Service, National Seed Storage Laboratory, Fort Collins, CO 80523, USA

(C) C.A.B International, 1993 